\title{
研究業績 (欧文)
}

\section{Semeia-Quelle und Dualismus}

\section{im Johannesevangelium}

\section{Takashi Ohnuki}

1. Das Problem. Meine Aufgabe in diesem Aufsatz sehe ich darin, das Zusammentreffen der Semeia-Quelle (=SQ) mit dem johanneischen Dualismus, besonders mit dem ethischen Dualismus als eines seiner Bestandteile, so redaktionsgeschichtlich wie möglich $z u$ erklären. Zu diesem $Z$ weck setze ich unten zwei der neuesten Forschungen zu diesem Problem, J. Becker, Wunder und Christologie, NTS 16, 1969/70 S. 130-148 und W. Nicol, The Semeia in the Forth Gospel, Leiden 1972 als Gegenstand einer kritischen Auseinandersetzung voraus. Der erstere untersucht hauptsächlich die theologischen Seiten des Problems, aber behandelt ihre redaktionsgeschichtliche Beziehung zur historischen Situation des Johannes und seiner Gemeinde nicht. Der letztere dagegen nimmt zwar Rücksicht auf diese Situation als den "Sitz im Leben" der johanneischen Redaktion, beachtet aber den Dualismus nicht genug.

2. Quellenkritik. Daß die SQ-Hypothese als ganze gültig sei, hat schon eine breite Anerkennung gefunden, obwohl es in bezug auf den quantitativen Umfang der Quelle noch bestreitbar ist: Es ist mehr als wahrscheinlich, daß SQ keinen ethischen Dualismus enthielt, der aus einem Verspaar von "Licht (Tag)" und "Finsternis (Nacht)" bestand: In 9, 1-8 und 11, 5-16 z. B., wo der Dualismus den Zusammenhang einer auf SQ zurückgehenden Wundergeschichte zerstört, sind die Vers- 
paare von "Tag (Licht)" und "Nacht" (9, 3b-5;11, 8-10) unverkennbar Einschiebsel des Johannes. Das bestätigen besonders

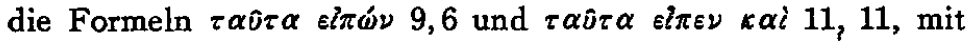
denen er wieder den zerbrochenen Zusammenhang der SQ aufnimmt. (vgl. 11, 28) Nicht nur der ethische Dualismus, sondern die dualistische Vorstellung überhaupt muß wohl dieser Quelle ganz fremd gewesen sein.

Der ethische Dualismus findet sich sonst auch im Prolog (besonders V. 4f. 9); 3, 19; 8, 12 und 12, 35f. 46. Dem Prolog liegt vielleicht eine schriftliche Vorlage zugrunde. Bei den tubrigen Stellen muß man aber eher eine freie Tradition, die auch in das johanneische Denken tief eingedrungen ist, als eine schriftliche Quelle (z. B. Bultmanns "Offenbarungsreden-Quelle") annehmen, weil sie alle ihrer Form und ihrem Inhalt nach eine Art Sprichwort darstellen, das auch ohne schriftliche Fixierung leicht umlaufen kann.

3. Formgeshichtliche und traditionsgeschichtliche Kritik. Formgeschichtlich gesehen ist $\mathrm{SQ}$ eine Sammlung von Wundergeschichten. Es fehlt ihr, worauf J. Becker mit Recht hingewiesen hat, nicht nur Dualismus, sondern auch Ekklesiologie, mythischer Rahmen der Christologie und Eschatologie. Allerdings muß man sich davor huten, dieser Quelle eine Eschatologie völlig abzuerkennen, denn Jesus wird in 6, 14 mit dem eschatologischen Propheten identifiziert. Trotzdem sind alle Wunder Jesu in SQ ihrem Wesen nach nicht die den Anfang der endzeitlichen Herrschaft Gottes beweisenden eschatologischen Wunder wie in der Q-Überlieferung (vgl. Mt 11, 2-6), sondern Epiphaniewunder des Sohnes Gottes (20, 30f). In dieser Eigenart des Wunderbegriffes gibt es keinen Unterschied zwischen SQ und der vormarkinischen Wundertradition. Bei beiden tritt die Wirklichkeit des Wunders anstelle des theologischen Denkens in den Vordergrund. Genauer gesehen ist sie jedoch in 
SQ aberbetriebener als in der letzteren. Das bedeutet, daß SQ traditionsgeschichtlich einer jüngeren Schicht der urchristlichen Wundertradition angehört.

In der ältesten Schicht der Tradition wurden m. E. die einzelnen Wundergeschichten getrennt überliefert und schlossen wohl keinen eindeutig christologischen Titel ein. Vom Standpunkt dieser ältesten Traditionsschicht her betrachtet ist es also gar nicht selbstverständlich, daß SQ ihre Wundergeschichten mit einer Reihenfolge versehen und sie alle unter dem christologischen Titel, “dem Sohn Gottes", zusammengezogen hat. In der Geschichte der urchristlichen Wundertradition markiert $\mathrm{SQ}$, in der die älteren, mehr oder weniger vereinzelt überlieferten Wundergeschichten nach einer bestimmten christologischen Idee, die ihnen ursprünglich fremd gewesen sein muß, ausgewählt und organisiert wurden, qualitativ eine neue Stufe. Und der Zweck der Auswahl und Organisation in SQ ist ihrem Selbstzeugnis $(20,30 \mathrm{f})$ nach in ihrem "Sitz im Leben", d. h. in einer auswärtigen Mission ihrer Träger zu suchen.

SQ besitzt daher eine Christologie, ja eine Herrlichkeitschristologie, obwohl es ihr an theologischen Vorstellungen wie den oben genannten mangelt. In ihr muß sich die Herrlichkeit Jesu monistisch durch alle Wundergeschichten gezogen und fast immer die "Akklamation" der Zuschauer gefunden haben, denn die Ablehnung seitens der "Pharisäer (od. Juden)" wurde erst von Johannes eingefügt. $\mathrm{Da}$ die "Aikklamation" der Zuschauer nicht für die älteste, sondern für die jüngste Form der Wundergeschichte konstitutiv ist, kommt SQ auch in dieser Hinsicht dem Idealtypus der Wundergeschichte nahe. (Bultmanns "Wundergeschichte" sowohl als Dibelius' "Novelle" sind m. E. in ihrer methodischen Eigenart ein idealtypisches Gebilde. Solch idealtypischer Zug ist, um es genauer zu sagen, beim letzteren stärker als beim ersteren.) 
Der ethische Dualismus geht traditionsgeschichtlich nicht direkt auf den gnostischen Vorstellungsbereich, sondern zunächst auf dieselbe Traditionsströmung zurück, die auch im Neuen. Testament selbst hin und da (Röm 13, 11-14; 2Kor 6, 14; Eph. 5, 8f; 1Th 5, 5f) zum Vorschein kommt. Und diese Tradition als ganze geht vielleicht noch weiter auf die spätjüdische Apokalyptik (z. B. Die Testamente der 12 Patriarchen und 1QS 3 , 13ff; 1QM usw.) und Weisheitsliteratur zurück.

4. Redaktionsgeschichichtliche Kritik. Was den "Sitz im Leben", d. h. die historische Situation des Johannes als Redaktor und seiner Gemeinde, hauptsächlich markiert, ist nicht eine Auseinandersetzung mit Gnostizismus, sondern der feindliche Streit mit dem gleichzeitigen pharisäischen Judentum. Dieses hat sich gegen die Verkündigung des Erhöhten (=des Menschensohnes) von der johanneischen Gemeinde abgeschlossen. Das bezeugt vor allem 3, 11ff, wo eine polemische Anklage des Johannes gegen die Pharisäer in seiner Gegenwart in den Zusammenhang des. Dialogs zwischen Jesus und Nikodemus anachronistisch eingedrungen ist. Diese Situation, in der die johanneische Gemeinde sich befand, muß der Herrlichkeitschristologie und dem "Sitz im Leben" (d. h. einer auswärtigen Mission) der SQ widersprochen haben. Der Herrlichkeit Jesu, die sie monistisch durchzog, muß jetzt Johannes irgendeine Schranke setzen. Es verhält sich nicht anders mit der Universalitătder Gottesliebe zur Welt, deren Vorstellung er als Tradition aufgenommen haben muß ( $\mathrm{ggl} .3,16$ ).

Die Lösung, welche Johannes diesen Widersprüchen gegeben hat, ist eine Art Theodizee. Auf der einen Seite nämlich billigt er den Missionswillen der SQ und die Tradition vom universalen Heilswillen Gottes, indem er die Wundergeschichten der Quelle nur in die erste Hälfte seines Evangeliums aufnimmt und Jesus in seiner Herrlichkeit trotz der Ablehung 
seitens der "Juden" durchweg als "das Licht der Welt" (3, $19 ; 8,12 ; 9,5 ; 11,9 ; 12,35 f .46)$ darstellt. Auf der anderen Seite aber sondert er ihn in der Abschiedsrede als das von der Welt abgelehnte Licht eng in die Jüngergemeinde ab. Damit klagt er vor allem das gegenwärtige pharisäische Judentum an, es sei wegen seiner Ablehnung der Verkündigung seiner Gemeinde schon jetzt von der Offenbarung der Herrlichkeit Jesu ganz ausgeschlossen. Die johanneische Gemeinde ist ihrem Selbstverständnis nach eine Versammlung von Individuen, die sich mit dem erhöhten Jesus in dex Offenbarung durch den Parakletos persönlich verbindet. Von dieser Offenbarung aus betrachtet deuten alle Worter und Werke des irdischen Jesus bereits auf die Herrlichkeit des erhöhten Jesus hin. Die Welt, die von dieser Offenbarung ausgeschlossen ist, kann also nicht die Herrlichkeit des Wunders Jesu sehen. Das widerspricht aber nicht der ursprünglichen Universalität des Heilswillen Gottes, denn es ist nur eine Konsequenz der Entscheidung der Welt gegen die Verkündigung der Gemeinde (vgl. 3,16-21;12, $35-43 ; 14,22-24$ ).

In dieser theologischen Absonderung der Herrlichkeit Jesu und der Gemeinde von der Welt besteht der johanneische Dualismus. Er ist eine radikale und einheitliche Sinndeutung, die Johannes und seine Gemeinde in ihrer historischen Situation über ihr Selbst und die Welt entwickelt haben. Eben in ihrer intellektualistischen Eigenart hat das johanneische Denken eine allgemeine Verwandtschaft mit dem gnostischen. Das Verspaar von "Licht" und "Finsternis", das der Tradition des ethischen Dualismus entstammt, ist nichts weiter als eines von vielen Begriffen, die der Sinndeutung ebenso dienen könnten.

Dem entspricht literarisch, daß Johannes sein Evangelium durch seinen redaktionellen Sammelbericht $12,35-43$ in zwei große Teile, d. h. "das Buch des Zeichens" und die Abschieds- 
rede eingeteilt hat. In ersterem haben die Wundergeschichten der SQ einen christologischen Sinnwandel durchgemacht. Die Herrlichkeit des irdischen Jesus in seinen Wundern in SQ ist nämlich jetzt im Hinblick auf die Herrlichkeit des Erhöten reinterpretiert worden. Und das hat auch dazu gefuhrt, daß die Wirklichkeit der Wunder Jesu ungemein zweideutig geworden ist, und daß die johanneische Christologie als ganze auch von demselben zweifachen Standpunkt beherrscht ist, den wir oben in 3,11ff festgestellt haben.

Bevor SQ Eingang in "das Buch des Zeichens" fand, hatten einige von ihren Wundergeschichten, wie die in Kap., 5,6 und 9 des jetzigen. Evangeliums, sich $z \mathfrak{u}$ "erweiterten Apophthegmata", wie der Beelzebul-Streit in der Q-Überlieferung (Mt 12,22-30 Par.), verändert. Mit diesem Formwandel ging auch ihr Funktionswandel einher: Diese "erweiterten Apophthegmata" dienten nicht mehr einer auswärtigen Mission wie .SQ, sondern einer Predigt in der Gemeinde, die starke Apologetik und Polemik gegen das pharisäische Judentum leistete. Das Evangelium des Johannes selbst, welches solche Predigten einschließt, wurde geschrieben, um die einzelnen Glieder der Gemeinde ins ewige Leben zu führen.

Aber unbeantwortet bleibt noch die redaktionsgeschichtlich entscheidendere Frage: Warum ein Evangelium? Um diese Frage zu beantworten, müBte ich die Passions- und Ostergeschichte behandeln, was aber über die Aufgabe dieses Aufsatzes hinausgeht. 